\title{
Politique et alimentation : l'allaitement, une préoccupation ancestrale du pouvoir
}

\section{Emmanuelle ROMANET}

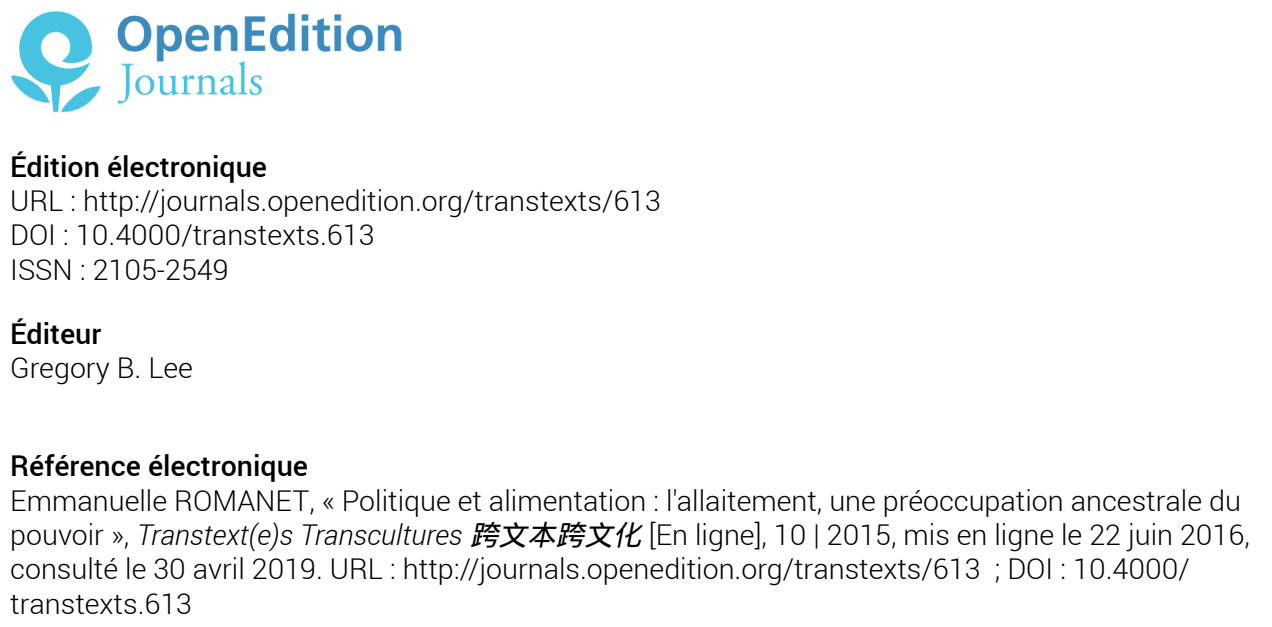

Ce document a été généré automatiquement le 30 avril 2019

(C) Tous droits réservés 


\title{
Politique et alimentation : l'allaitement, une préoccupation ancestrale du pouvoir
}

\author{
Emmanuelle ROMANET
}

1 Si l'Etat ne s'est intéressé que récemment à l'alimentation de la population (à travers le célèbre « Cinq fruits et légumes par jour », par exemple), il y a cependant un domaine de l'alimentation dans lequel l'Etat a, très tôt, voulu intervenir : c'est l'alimentation des nourrissons. En effet, l'allaitement a toujours occupé une place particulière dans les questions d'alimentation.

\section{Le lait et le pouvoir}

2 Le lait maternel est un aliment à part. Dans l'Ancien Testament, le lait (et le miel) symbolisent la Terre promise. ${ }^{1}$ Le lait est avec le sang et le sperme une des grandes humeurs fondamentales du corps humain. Il est donc toujours chargé d'une haute valeur symbolique. D'ailleurs dans de nombreuses cultures, l'allaitement met en jeu les pouvoirs $\mathrm{du}$ lait et confère à certains nourrissons un destin singulier. C'est le cas de certains dieux ou héros de la mythologie antique comme les célèbres Romulus et Rémus, allaités par une louve. On sait (les ethnologues le confirment) qu'une parenté symbolique se construit grâce au lait, interdisant les mariages entre ceux qui ont été allaités par la même femme. ${ }^{2}$

3 La manière dont le corps fabrique le lait est restée longtemps mystérieuse. D'où des explications très variées et d'une grande richesse symbolique. En Occident, depuis l'Antiquité, on considère le lait comme du sang cuit et blanchi : le foetus se nourrit du sang maternel in utéro puis, après l'accouchement, le sang « monte » dans les seins de la mère où, après une « cuisson » (appelée « coction ») il se transforme en lait. Il y a donc continuité biologique entre la grossesse et l'allaitement. Et c'est sur cette continuité que les médecins, de l'Antiquité jusqu'au XIXe siècle, vont s'appuyer pour inciter à l'allaitement maternel. ${ }^{3} C^{\prime}$ est bien cette continuité qui est encore implicite, au début du 
XXe siècle, dans la maxime préférée du grand accoucheur Adolphe Pinard: « le lait de la mère appartient à l'enfant ${ }^{4}{ }^{4}$

4 Le lait est considéré comme un transmetteur. Ainsi, on croyait que par le lait, les qualités physiques et morales de la mère ou de la nourrice passaient à l'enfant. L'idée selon laquelle le lait absorbé véhiculait les caractères ou les propriétés de qui en était l'agent est récurrente. Le lait transmettrait ainsi vices ou qualités. Stendhal écrit d'ailleurs en 1837 que : "Cet ensemble d'habitudes et de manière de voir qui vous émerveille dans votre enfant et que vous appelez son caractère, lui est donné d'abord par sa nourrice ... ».5 C'est pour cette raison que le médecin Soranos d'Ephèse conseille dans son ouvrage de choisir une nourrice « sensible, vigilante et paisible». Ce livre constitue la somme de tout le savoir antique sur cette question. Et, on retrouve ces idées,à quelques détails près, dans de très nombreux ouvrages médicaux jusqu'au milieu du XIXe siècle. ${ }^{6}$

6 Au XIXe siècle, les médecins ne croient plus que le nouveau-né ingère avec le lait de sa nourrice les défauts et qualités de cette dernière. Mais tous ne semblent pas convaincus. Ainsi, en 1836, dans un des multiples ouvrages destinés à aider les jeunes mères à choisir une nourrice, on s'interroge :

7 «Peut-on choisir pour élever un enfant une nourrice bornée et peu intelligente ? Non, car son lait est bête, il pourrait épaissir l'intelligence du nourrisson. ${ }^{7}$ ”

8 Tout passe dans le lait, y compris les affections morales. Comme nous l'explique un dictionnaire de médecine usuelle et domestique de 18598: " Les troubles de l'âme n'agissent pas seulement sur la sécrétion du lait, ils altèrent également ses qualités « au point de changer sa couleur et sa saveur." Alphonse Daudet explique dans "Les Nounous » (1889) que " Quand nounou pleure (...) les sangs tournés font le lait aigre. » ${ }^{9} \mathrm{La}$ Baronne de Staffe dans son célèbre ouvrage intitulé «La maitresse de maison » écrit en 1892: «Les émotions morales de la nourrice sont nuisibles à l'enfant, il faut les lui éviter. $»^{10}$

9 Il y a aussi un lien entre l'alimentation de la femme qui allaite et la qualitéquantité du lait. Il y a donc des aliments à éviter comme le chou, la viande fumée, les épinards, l'ail, l'oignon ... L'alcool est, déjà à cette époque, à consommer avec modération. Et, bien entendu, il faut bien (au sens de beaucoup) manger quand on allaite. La nourrice est donc bien nourrie voire très bien. D'ailleurs, il existe une locution populaire : « Le morceau de la nourrice » qui signifie le meilleur morceau. ${ }^{11}$

Il faut aussi noter le lien existant entre allaitement et rapports sexuels : ces derniers sont considérés comme altérant le lait. Le tabou interdisant les rapports sexuels pendant l'allaitement a longtemps persisté.

$11 \mathrm{Au}$ XIXe siècle, on est aussi très vigilant sur la santé de la femme qui allaite. Elle ne doit avoir aucune maladie. La maladie passe dans le lait, comme la syphilis qui est très redoutée, à l'époque, par les familles aisées qui cherchent une nourrice sur lieu. ${ }^{12}$

Toutes ces particularités qui concernent le lait donnent aux médecins une compétence qu'ils revendiquent comme conseillers auprès des mères dans le choix d'une nourrice.

Le lait maternel est donc un aliment à part. On peut parler d'une véritable apologie de l'allaitement maternel, et ce, depuis fort longtemps. ${ }^{13} \mathrm{Au}$ XIXe siècle, les médecins incitent les femmes à allaiter. Tous les articles des dictionnaires médicaux prônent l'allaitement maternel. Aux avantages physiques, ils ajoutent des avantages moraux et sociaux : comme la piété filiale, ... sans oublier les bienfaits pour la santé de l'enfant. 
Le lait maternel, cet aliment à part, a donc toujours intéressé le pouvoir. La législation concernant l'allaitement est très ancienne. Le code d'Hammurabi, texte juridique babylonien daté d'environ 1750 avant J.C., statuait déjà sur l'allaitement : une nourrice qui n'allaite pas assez un nourrisson qui lui est confié, et cause sa mort, aura son sein tranché. ${ }^{14}$

En France, le premier texte officiel est l'Edit du roi Jean, en 1350. Il fixe le salaire des nourrices et les conditions. Il y a une multiplication de lois au XVIIIe siècle ayant pour but de protéger les nourrissons. Décrets, lois, ordonnances réglementent de plus en plus précisément "l'industrie » des nourrices dans une France, qui au XVIIIe siècle, a massivement recours à cette pratique.

En $1715^{15}$, Louis XIV prend une ordonnance qui interdit aux nourrices, en cas de grossesse ou de maladie, de prendre des enfants pour les allaiter, sous peine du fouet et d'amende. La grossesse et la maladie étant considérées comme un empêchement à la nourriture de l'enfant. Une ordonnance de 1727 défend aux nourrices d'allaiter deux nourrissons à la fois sous peine d'amende pour les maris et du fouet pour les femmes. On pourrait continuer ainsi $(1757,1762$...). Cette réglementation est rassemblée en 1781 dans un "Code des nourrices ${ }^{16}$, qui contient toutes les dispositions qui seront développées au siècle suivant.

Sous la Révolution, les choses n'évoluent guère. Un décret de la Convention de juin $1793^{17}$ stipule que «les secours ne seront apportés qu'aux mères qui allaiteraient leurs enfants » ${ }^{18}$. Beaucoup de familles ont, d'ailleurs, du mal à payer les frais de " nourriture » de leurs bébés. Et au XVIIIe siècle, des quêtes sont faites dans toutes les paroisses de Paris pour le paiement des mois de nourrices. Certains princes contribuent aussi par leur générosité.

Le pouvoir, quand il ne légifère pas, montre son intérêt pour la « nourriture » des jeunes enfants en faisant des dons destinés à aider les familles indigentes qui ne peuvent payer les nourrices. En 1810, à l'occasion de son mariage, l'Empereur par un décret règle les dettes contractées par des parents de Paris et du département de la Seine envers les nourrices..$^{19}$ En 1821, Le roi Louis XVIII, à l'occasion de la naissance du duc de Bordeaux, fait la même chose.

\section{L'allaitement à travers la politique de la petite enfance}

19 En France, au XIXe siècle, l'Etat s'empare de la question de l'allaitement à travers la politique de la petite enfance. La mise en nourrice a alors une conséquence directe sur la mortalité infantile qui est très élevée. Les chiffres de la surmortalité des enfants placés en nourrice sont, en effet, édifiants. Pour preuve, ces chiffres issus d'un mémoire de 1866 que le ministre de l'Instruction publique, Victor Duruy, a communiqué à l'Académie de médecine. Ce mémoire est l'œuvre du Docteur Monot, médecin dans la Nièvre. Il décrit le vide laissé dans les villages par le départ des femmes venues se placer comme nourrice à domicile à Paris. Entre 1858 et 1864, sur les 2884 femmes ayant accouché dans son canton, les deux tiers (1897) ont émigré. ${ }^{20}$

Le Docteur Monot évoque la mort massive des enfants nouveau-nés laissés. Et le sort des «Petits Paris », ce sont les nouveau-nés de Paris en nourrice dans le canton, est encore pire : le taux de mortalité atteint $71 \%$ pendant la première année de leur existence. ${ }^{21} \mathrm{Le}$ taux de mortalité infantile pour l'ensemble de la France à cette époque est de 17,9\%.22 
21 Les médecins et les moralistes s'emparent donc de cette question et critiquent la mise en nourrice. $1860 .^{29}$ moyennant salaire, en nourrice, (...) hors du domicile de ses parents. Il devient, par ce fait, l'objet d'une surveillance de l'autorité publique, ayant pour but de protéger sa vie et sa santé. $»^{30}$ Cette surveillance est assurée par le préfet assisté de comités départementaux et de Commissions locales. Des inspecteurs sont nommés par les préfets pour une surveillance constante des nourrissons. Tout placement d'enfant en nourrice 
doit être déclaré, etc. ... Obligation est faite aux nourrices de demander certificats ou autorisation d'exercice. Un carnet est mis en place. Il renseigne sur la nourrice, le bébé, les paiements, les visites médicales ... Il contient des recommandations de l'Académie de médecine, comme : «Pendant la première année, la seule nourriture de l'enfant doit être le lait »

Les enfants concernés par la loi dépendent, localement, du service de la Protection des enfants du premier âge. La loi prévoit aussi des inspections médicales chez les nourrices à la campagne par des médecinsinspecteurs ${ }^{31}$, une à 2 fois par an. Ces derniers doivent rédiger des rapports annuels ${ }^{32}$. La loi Roussel, il faut le souligner, ne surveille que les enfants placés en nourrice à l'extérieur du domicile des parents. Elle exclue ainsi toute surveillance des nourrices sur lieu qui travaillent au domicile des familles aisées. Cette loi ne s'applique donc qu'à une partie de la population, la plus démunie : les familles pauvres des villes et les familles de paysans. La surveillance porte sur ce que l'on appelle alors «les classes dangereuses» mais pas sur les familles dites "honorables». La loi exerce alors un contrôle social. ${ }^{33}$ Cette loi comporte, enfin, un aspect statistique: les départements doivent envoyer à Paris des données tous les ans afin d'évaluer l'impact de la loi.

31 La question de l'alimentation du petit enfant a donc pris un tournant au XIXe siècle. C'est devenu une affaire d'hommes : médecins, législateurs, hommes politiques ...

\section{Application de cette politique à Lyon au $\mathrm{XIX}^{\mathrm{e}}$ siècle} politique.
C'est à travers l'exemple de Lyon, que nous étudierons la mise en place d'une telle

Lyon, pendant tout le XIXe siècle, demeure en effet la ville de France qui envoie la plus forte proportion de ses nouveaunés en nourrice. Plus de la moitié des bébés sont confiés à des nourrices « à emporter » (à la campagne), sans compter les nourrissons pourvus d'une nourrice "sur lieu» (au domicile des parents). Lyon est donc un cas particulier: l'allaitement y est mercenaire. Selon les chiffres du ministère de l'Intérieur, $47 \%$ des enfants nés à Lyon en 1897 sont mis en nourrice à l'extérieur, contre $32 \%$ pour Paris. ${ }^{34} \mathrm{Et}$ sur les 6000 enfants placés en nourrice par an, 4000 décèdent. ${ }^{35}$

Localement, les agents d'exécution de la loi Roussel sont les préfets, assistés de Comités départementaux de protection des enfants du premier âge. Les sociétés protectrices de l'enfance et autres associations sont intégrées dans ces comités.

Ces associations privées ont des principes moraux, catholiques et bourgeois. Elles auraient souhaité que leur soit confiée l'exécution de la loi Roussel. Mais les fonctionnaires et les législateurs ne veulent pas que les associations privées se substituent au service public, par souci de laïcisation.

A Lyon, l'Etat s'est beaucoup appuyé sur les œuvres déjà existantes, qui s'occupaient de la petite enfance. Il faut dire que dans cette ville, il y a beaucoup d'œuvres de bienfaisance. Dès les années 18301840, la ville a le titre de «ville des bonnes œuvres » ou de «capitale de la charité » : le patronat lyonnais considérant l'assistance comme un devoir. ${ }^{36}$ Lyon est aussi appelé le « Manchester français ${ }^{37}$ ».

Ce sont donc des œuvres privées mais l'Etat agit via ces œuvres, qu'il subventionne. D'ailleurs, parmi les agents qui exécutent la loi Roussel, on trouve des administrateurs de 
la Société de la Charité Maternelle de Lyon et des administrateurs de la Société Protectrice de l'Enfance de Lyon, les deux principales œuvres dédiées à ces questions.

Les Sociétés de Charité maternelle ont pour but de favoriser l'allaitement par la mère. Elles sont reconnues et admises à recevoir des subventions de l'Etat, des départements ou municipalités. A Lyon, la société a été créée en $1786 .{ }^{38}$ Elle compte environ 500 adhérents. ${ }^{39}$ Elle aide des mères dans l'indigence et les encourage, financièrement, à nourrir ellesmêmes leur enfant mais à condition que ce dernier soit légitime. ${ }^{40}$ Non seulement les mères doivent être mariées mais elles doivent aussi fournir un certificat de bonnes mœurs ${ }^{41}$ La société a de bonnes relations avec les pouvoirs publics ${ }^{42}$. Elle est de loin la plus subventionnée avec des subsides publics qui représentent 30\% de ses ressources. ${ }^{43}$ Mais à partir de 1880, elle perd sa subvention municipale et voit celle octroyée par l'Etat baisser considérablement. Les Républicains l'accusent de conditionner ses aides au mariage religieux des mères secourues et souhaitent laïciser l'action des œuvres. ${ }^{44} \mathrm{En}$ 1836, elle aidait 174 mères ; en 1872, la Société en a secouru 1150.45

La Société Protectrice de l'Enfance de Lyon, elle, est fondée en $1866^{46}$ sur l'initiative d'un chirurgien lyonnais ${ }^{47}$. Ce médecin s'est ému de l'importante mortalité des enfants placés en nourrice ${ }^{48}$. C'est une œuvre nouvelle. Elle n'a donc pas que des objectifs éducatifs et moralisants et elle se dégage de la tutelle catholique. Ses objectifs sont plutôt médicaux et sanitaires. Elle est sous la tutelle d'éminents médecins lyonnais ${ }^{49}$. Cette œuvre préfigure la dimension hygiéniste qui se développe en France à la fin XIXe siècle. ${ }^{50}$ Elle est reconnue d'utilité publique et entend lutter contre la trop forte mortalité infantile en facilitant l'allaitement maternel dans les milieux urbains. Pour ce faire, elle distribue des prix aux mères et nourrices méritantes. Elle développe aussi un réseau de crèches..$^{51}$ Quatre crèches sont créées en 1871. En 1883, il y en a neuf. ${ }^{52}$ Les crèches en milieu urbain évitent la mise en nourrice à la campagne. Elles « incitent » à l'allaitement maternel en offrant la gratuité de la garde si les mères s'engagent à venir allaiter leur enfant deux fois par jour. ${ }^{53}$ Dans un premier temps, les pouvoirs publics se déchargent sur l'assistance privée pour développer les crèches. Mais, au tout début du XXe siècle, les crèches passent à la charge de la municipalité.

40 A Lyon, entre 1870 et 1914, neuf sociétés se créent autour de la maternité et des soins aux nourrissons. Cinq de ces œuvres font de la consultation de nourrissons qui ont pour but d'encourager les mères à allaiter. C'est le cas l'CEuvre des Poupons des Brotteaux ${ }^{54}$ ou encore de l'€Euvre des Nourrissons des Terreaux. ${ }^{55}$

Dans cette ville, en 1887, 1500 à 2000 enfants légitimes sont secourus. ${ }^{56}$

Ces sociétés (celles que nous venons d'évoquer; mais aussi d'autres comme la Société d'Encouragement au nourrissage maternel, par exemple) sont animées par des Comités de dames, parmi lesquelles se retrouvent les grands noms de la haute société lyonnaise ${ }^{57}$. Il y a donc un mélange de préoccupations "sociales et hygiénistes" (préoccupations de l'Etat) et un souci "de reconquête morale et religieuse de milieux en perdition..$^{58}$ " (préoccupations des élites locales). Au XXe siècle, les institutions publiques républicaines prennent le relais des œuvres de charité. Mais à Lyon, la substitution est plus lente qu'ailleurs, il faudra attendre l'entredeuxguerre. ${ }^{59}$

Lyon est connue pour sa gastronomie et ses restaurants. La ville fut aussi la première en France à créer un restaurant municipal gratuit pour les mèresnourrices. Il fut décidé en 1910 par Edouard Herriot, le maire de Lyon $^{60}$ : le pouvoir municipal souhaitant encourager ainsi l'allaitement maternel. Car pour bien allaiter son enfant, il faut bien se 
nourrir, et ce restaurant est donc réservé aux femmes enceintes (dès le cinquième mois de grossesse) et aux mères qui allaitent ellesmêmes leurs enfants. ${ }^{61}$

\section{Conclusion} gouvernement mettra en place des programmes de distribution de lait à l'école : le célèbre verre de lait voulu par Pierre Mendès France (dès 1954)! Afin de pallier les carences alimentaires de l'après-guerre, de faire de l'éducation nutritionnelle mais aussi de développer l'économie laitière.

\section{NOTES}

1. Yvonne Knibiehler, «L'allaitement et la société » in Recherches féministes, vol.16, 2003, p.12.

2. Françoise Héritier, Les deux sœurs et leur mère. Anthropologie de l'inceste, Paris, Odile Jacob, 1994 (2012), p. 274.

3. Un célèbre pédiatre, le Docteur Bouchut dans son traité de 1862 écrit que « le lait est un dérivé du sang. ». Dr

E. Bouchut, Hygiène de la petite enfance, Paris, JB Baillière, 1862, p. 143144.

4. Catherine Rollet, "Histoire de l'allaitement en France: pratiques et représentations", www.santeallaitementmaternel.com, novembre 2005.

5. Stendhal, Voyage à Lyon (1837), Christian Pirot éditeur, 1995, p.21.

6. Yvonne Knibiehler, «L'allaitement et la société », op. cit., p. 15.

7. Pierre Maigne, Choix d'une nourrice, Paris, Crochard, 1836, p. 201.

8. Bayle et Gibert, Dictionnaire de médecine usuelle et domestique, Paris, M. du Closel, 1859, p.98.

9. Alphonse Daudet, « Les Nounous », 1889, in La jeune mère, septembre 1889, p. 134. 
10. Baronne de Staffe, La maîtresse de maison, Paris, V. Havard, 1892, p. 187.

11. Fanny FaÿSallois, Les nourrices à Paris au XIXe siècle, Paris, Payot, 1997, p. 199.

12. Nourrices contaminées à Lyon 18821906 (ADR 3X 1927 à 3X 1931)

13. Fanny FaÿSallois, Les nourrices à Paris au XIXe siècle, op. cit., p. 115.

14. Paragraphe 194.

15. Le 29 janvier 1715.

16. Les nourrices subissaient une visite médicale, ne pouvaient avoir la garde de plus d'un enfant...

17. Du 28/06/1793.

18. Titre I, article 27.

19. "A l'occasion de son mariage, l'Empereur, par un décret du 25 mars 1810, fit remise de toute la dette pour mois de nourrice contractée envers le Bureau des nourrices par des pères et mères de la ville de Paris et du département de la Seine. » Faulcon, Rapport au Conseil de Surveillance sur les modifications à introduire dans l'organisation de la Direction des nourrices, Paris, 30 mai 1850, p. 10.

20. Charles Monot, De l'industrie des nourrices et de la mortalité des petits enfants, Paris, 1867, p.36.

21. Charles Monot, De la mortalité excessive des enfants la première année de leur existence, ses causes et des moyens de la restreindre, Paris, 1872, p.27.

22. Chiffres de 1865. Jacques Gélis, Mireille Laget et Marie Morel, Entrer dans la vie. Naissances et enfances dans la France traditionnelle, Paris, Gallimard, 1978, p. 186.

23. Fanny FaÿSallois, Les nourrices à Paris au XIXe siècle, op. Cit., p. 67.

24. Catherine RolletEchalier, La politique à l'égard de la petite enfance sous la IIIe République, INED PUF, 1990. p.5859.

25. «On ne veut plus de nombreuses familles. C'est là (...) une affaire de mœurs et la loi n'y peut rien » a ton même entendu à l'Assemblée Natioanle en 1874, in Durangel, Rapport ... Roussel, Assemblé Nationale, séance du 9 juin 1874, p. 180.

26. "... toute mère qui confie un enfant à une nourrice mercenaire double et triple volontairement les chances de mort de cet enfant. » in Brochard, De l'allaitement maternel du point de vue de la mère, de l'enfant et de la société, Paris, 1874, p. 119.

27. Docteur Brochard, Almanch illustré de la jeune mère, 1876, p.13.

28. Devilliers, Nouveau dictionnaire de médecine et de chirurgie, article " Nourrices ", 1877 et Delore, Dictionnaire encyclopédique des Sciences médicales, article " Nourrices », 1879 et Le Fort, «De la mortalité des enfants et de l'Industrie des nourrices en France », 1870 in Fanny FaÿSallois, Les nourrices à Paris au XIXe siècle, op. Cit., p. 72.

29. Ministère de l'Intérieur, Enfants assistés, Enquête de 1860, p.163.

30. Fanny FaÿSallois, Les nourrices à Paris au XIXe siècle, op. cit. p. 88.

31. ADR 3X1885 Organisation, nomination des médecins inspecteurs dans le département du Rhône.

32. Ce service est assimilé au service des Enfants assistés (créé par décret impérial du 19/01/1811). Le service de Protection des enfants du premier âge est un service à part entière mais il est administré par l'inspecteur du service des Enfants assistés. ADR 3X 1879 et 3X 1880 Rapports annuels de l'inspecteur 18791900.

33. Mais la loi Roussel dans son article 8 dit que: "Toute personne qui veut se placer comme nourrice sur lieu est tenue de se munir du certificat du maire de sa résidence indiquant si son dernier enfant est vivant, et constatant qu'il est âgé de 7 mois révolus, ou s'il n'a pas atteint cet âge qu'il est allaité par une autre femme... » La loi espère ainsi éviter la mort du nourrisson de la femme partie se placer comme nourrice. Cet aspect de la loi Roussel n'est pas appliqué. Un décret du 25 février 1877 rappelle cet article 8. Mais, il ne sera pas mieux appliqué !

34. Jacques Bertillon, « Du degré d'efficacité de la loi du 23 décembre 1874 » in Journal de la société statistique de Paris, 1902, tome 43, p. 325. 
35. D'après un article de FrançoisMarguerite Barrier, chirurgien de l'Hôtel Dieu de Lyon, daté de 1866 et intitulé

« Note sur les moyens d'améliorer les conditions des enfants en nourrice » in site d'enseignement d'Histoire de la médecine, Université Claude Bernard Lyon 1, Diaporama Michel David.

36. Bernadette Angleraud et Catherine Pellissier, Les dynasties lyonnaises Des MorinPons aux Mérieux, du XIXe siècle à nos jours, Paris, Perrin, 2003, p. 402.

37. Bernadette Angleraud, Lyon et ses pauvres Des cuvres de charité aux assurances sociales 18001939, Paris, L'Harmattan, 2011, p. 325.

38. Bernadette Angleraud et Catherine Pellissier, Les dynasties lyonnaises ..., op. cit. p. 593.

39. Bernadette Angleraud et Catherine Pellissier, Les dynasties lyonnaises ..., op. cit. p. 399.

40. Bernadette Angleraud et Catherine Pellissier, Les dynasties lyonnaises ..., op. cit. p. 593.

41. Bernadette Angleraud, Lyon et ses pauvres, op. cit. p. 44.

42. Bernadette Angleraud, Lyon et ses pauvres, op. cit. p. 78.

43. Bernadette Angleraud, Lyon et ses pauvres, op. cit. p. 86.

44. Bernadette Angleraud, Lyon et ses pauvres, op. cit. p. 140.

45. Rapport Roussel JO 26/07/1874.

46. Bernadette Angleraud et Catherine Pellissier, Les dynasties lyonnaises ..., op. cit. p. 593.

47. FrançoisMarguerite Barrier, chirurgien de l'Hôtel Dieu de Lyon. In Site d'enseignement d'Histoire de la médecine, Université Claude Bernard Lyon 1, Diaporama Michel David.

48. Il s'en explique dans un article de 1866 intitulé « Note sur les moyens d'améliorer les conditions des enfants en nourrice ». Il en est le président.

49. Les docteurs Rougier et Rodet.

50. On y retrouve des personnalités de confessions différentes, issues du monde médical et de l'industrie. Ex : François Gillet.

51. Bernadette Angleraud et Catherine Pellissier, Les dynasties lyonnaises ..., op. cit. p. 594.

52. Catherine RolletEchalier, La politique à l'égard de la petite enfance sous la IIIe République, op. cit. p. 530.

53. Un complément est assuré par du lait stérilisé. In Bernadette Angleraud, Lyon et ses pauvres, op. cit. p. 160.

54. Madame Gillet en est présidente (1ère année en 19061907) in Bernadette Angleraud et Catherine Pellissier, Les dynasties lyonnaises ..., op. cit. p. 403.

55. ADR 3X 1853.

56. Rapport de la Protection Rhône, 1886, p. 39.

57. Mme Gillet et Mme Riboud.

58. Bernadette Angleraud et Catherine Pellissier, Les dynasties lyonnaises ... op. cit. p. 594.

59. Bernadette Angleraud et Catherine Pellissier, Les dynasties lyonnaises ... op. cit. p. 595.

60. Il est situé au 78 rue Saint Georges: une photo conservée aux AML nous montre des femmes portant leurs nourrissons. AML 4FI_3733.

61. Catherine RolletEchalier, La politique à l'égard de la petite enfance sous la IIIe République, op. cit., p. 373.

62. John Ward, De l'enfant "sans état» à l'enfant comme "personne». L'évolution de la condition des enfants en France, du milieu du XIXe siècle aux années 1920, in www.editionsharmattan.fr 


\section{RÉSUMÉS}

Si l'Etat ne s'est intéressé que récemment à l'alimentation de la population (à travers le célèbre "Cinq fruits et légumes par jour », par exemple), il y a cependant un domaine de l'alimentation dans lequel l'Etat a, très tôt, voulu intervenir : c'est l'alimentation des nourrissons.

En effet, l'allaitement a toujours occupé une place à part dans les questions d'alimentation. Le code d'Hammurabi, texte juridique babylonien daté d'environ 1750 avant J.C., statuait déjà sur l'allaitement! Le lait est un aliment particulier, symboliquement chargé. Le pouvoir a donc cherché à légiférer, contrôler, organiser et favoriser l'allaitement.

En France, le XIXe siècle est caractéristique du souci de l'Etat dans ce domaine. Cette période est l'apogée de "l'industrie nourricière ", responsable d'une très forte mortalité infantile. L'Etat français a donc mis en place une politique à l'égard de la petite enfance dans laquelle l'allaitement tient une place importante. Cette politique s'adresse, bien entendu, tout particulièrement aux femmes, qu'elles soient mères ou nourrices.

Lyon est un bon exemple pour étudier la mise en place d'une telle politique. Cette ville demeure, durant tout le XIXe siècle, la ville de France qui envoie la plus forte proportion de ses nouveaunés en nourrice.

\section{AUTEUR}

\section{EMMANUELLE ROMANET}

Docteur en histoire, Emmanuelle ROMANET est chercheur associé à l'IETT (EA 4186), Université Jean Moulin Lyon 3. Elle travaille sur la situation des femmes dans leurs relations au monde du travail et la situation des femmes dans leur rôle de mère, de l'Ancien Régime jusqu'au XIXe siècle. 